\title{
EVALUACIÓN FORMATIVA A TRAVÉS DE METODOLOGÍAS ACTIVAS: PROYECTO RADIOEDUBU
}

Formative assessment through active methodologies: RADIOEDUBU

Formação de avaliação através de metodologias empréstimos: RADIOEDUBU

\author{
Vanesa Delgado Benito (1) \\ David Hortigüela Alcalá (2) \\ Vanesa Ausín Villaverde (3) \\ Víctor Abella García (4)
}

(1) Universidad de Burgos, España. Teléfono: +34 947111417. Correo electrónico: vdelgado@ubu.es

(2) Universidad de Burgos, España. Correo electrónico: dhortiguela@ubu.es

(3) Universidad de Burgos, España. Correo electrónico: vausin@ubu.es

(4) Universidad de Burgos, España. Correo electrónico: vabella@ubu.es

\begin{abstract}
Resumen
En los últimos tiempos, el Aprendizaje Basado en Proyectos (ABP) se ha mostrado como una metodología activa innovadora para el desarrollo de competencias en Educación Superior. En estas páginas se presenta un proyecto cuya finalidad ha sido la creación colaborativa de una radio (\#RadioEdUBU), por parte de estudiantes de diversos Grados de Educación de la Universidad de Burgos, mediante entrevistas en formato podcast realizadas a expertos sobre TIC y educación. Se han utilizado sistemas de evaluación formativa y compartida, tanto durante el proceso de realización del proyecto mediante grupos de trabajo como en el producto final. Todo ello a través de estrategias como la autoevaluación y la coevaluación y utilizando diversas rúbricas.

Palabras clave: aprendizaje basado en proyectos; metodologías activas; innovación educativa; evaluación formativa; enseñanza universitaria
\end{abstract}

\begin{abstract}
Recently, Project Based Learning (PBL) has been shown as an innovative and active methodology for the development of skills in Higher Education. In these pages we Evaluación formativa a través de metodologías activas: proyecto RADIOEDUBU
\end{abstract}


present a project whose purpose has been the collaborative creation of a radio (\#RadioEdUBU), by students of different Degrees in Education at the University of Burgos, through interviews in podcast format made to experts on ICT and Education. Using formative and shared evaluation systems, both during the realization of the project through working groups and in the final product. All this through strategies such as self-assessment, co-evaluation and various rubrics.

Keywords: project-based learning; active methodologies; educational innovation; formative assessment; higher education

\section{Resumo}

Nos últimos tempos, o Aprendizagem Baseada em Pprojetos (ABP) foi mostrado como uma metodologia activa para o desenvolvimento de habilidades inovadoras no Ensino Superior. Nestas páginas de um projeto cujo objetivo foi a criação colaborativa de rádio (\#RadioEdUBU), por estudantes de vários níveis de ensino da Universidade de Burgos, entrevistas em podcast com especialistas em TIC e formato de educação é apresentada. Os sistemas que utilizam a avaliação formativa e compartilhada, tanto durante o processo de implementação do projeto por meio de grupos de trabalho e do produto final. Tudo isso através de estratégias como a auto-avaliação e de avaliação pelos pares e usando diversas rubricas.

Palavras-chave: aprendizagem baseada em projeto; metodologias ativas; a inovação educacional; avaliação formativa; ensino universitário

\section{Introducción}

Las Tecnologías de la Información y la Comunicación (TIC) se muestran como un recurso innovador que puede facilitar el desarrollo de competencias en Educación Superior. Por ende, el aula universitaria se convierte en un excelente laboratorio donde poder realizar experiencias educativas centradas en el aprendizaje de los estudiantes y que permitan la participación activa de estos mediante la utilización de las TIC.

Esta comunicación presenta una experiencia de innovación docente a través del Aprendizaje Basado en Proyectos (ABP) como metodología activa (Ausín, Abella, Delgado \& Hortigüela, 2016) que permite el desarrollo de competencias en los estudiantes (Morales \& Torres, 2015). El ABP o PBL por sus siglas en inglés (Project- 
Based Learning) es un enfoque educativo que fomenta que los estudiantes "aprendan a aprender” y que trabajen de manera colaborativa en grupo para buscar soluciones a un problema real (Xu \& Liu, 2010). Además, les proporciona contextos de aprendizaje reales, implicándoles en el diseño, la resolución del problema y, sobre todo, en la toma de decisiones y la actividad investigadora que conlleva (Grahame, 2011).

Con el ABP se fomenta la participación de los estudiantes en los procesos de evaluación lo cual revierte en su implicación en el proceso de enseñanza-aprendizaje.

El objetivo del proyecto aquí presentado, realizado bajo la metodología ABP, ha sido la creación colaborativa de una radio educativa (\#RadioEdUBU) a través de entrevistas en formato podcast realizadas a expertos sobre TIC y educación. Durante el proyecto se han llevado a cabo diferentes procesos de evaluación formativa y compartida.

\section{Contextualización}

El proyecto \#RadioEdUBU se lleva realizando durante los últimos cuatros cursos académicos en la asignatura “TIC aplicadas a la educación”, incluida en el en el plan de estudios del Grado en Pedagogía, Grado de Maestro en Educación Primaria y Grado de Maestro en Educación Infantil de la Universidad de Burgos. Se trata de una asignatura de formación básica y con una carga total de 6 créditos ECTS para los estudiantes en las tres titulaciones mencionadas. Hasta la fecha, un total de 194 estudiantes han participado en el proyecto, dando como resultado 46 entrevistas en formato podcast.

\section{Diseño y desarrollo}

Para la realización del proyecto \#RadioEdUBU, se formaron grupos de trabajo de 4 o 5 estudiantes, los cuales siguieron las diferentes fases establecidas para su consecución (Figura 1).

1) Selección del tema relacionado con las TIC y la Educación.

2) Entrevista radiofónica en formato podcast.

3) Creación de un vídeo promocional de la entrevista utilizando la técnica de animación Cut out.

4) Elaboración de un sitio web para presentar el trabajo final.

5) Presentación y defensa oral de los grupos de trabajo. 
Como puede observarse en la figura anterior, en el proceso de realización los grupos de trabajo debían realizar diferentes entregables (E1, E2, E3, E4, E5 y E6) para cada una de las fases. Además, durante el transcurso del proyecto, cada grupo realizó un blog como portfolio donde relataban sus progresos.

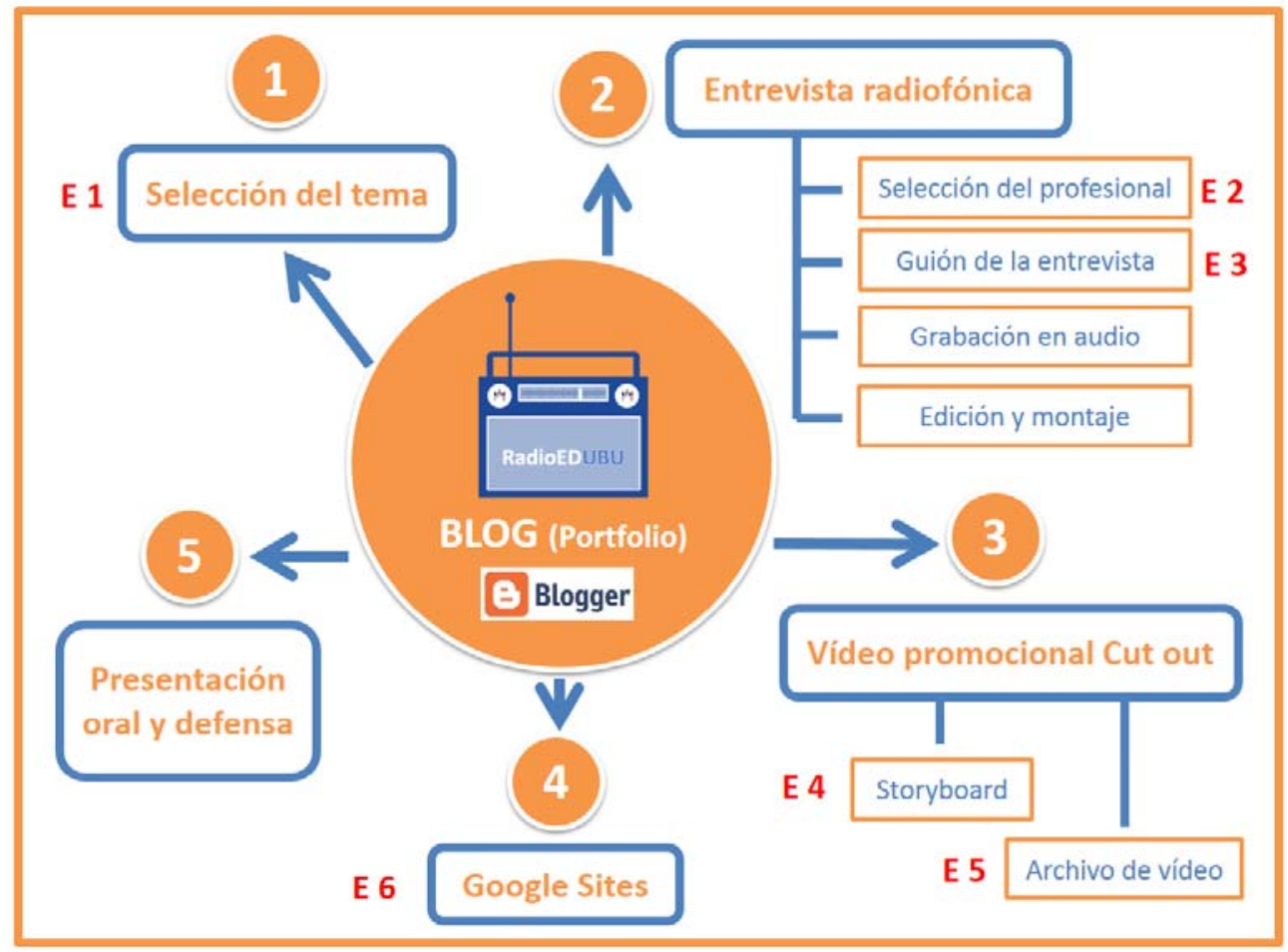

Figura 1.

Esquema del proyecto \#RadioEDUBU

\section{Evaluación}

Durante todo el desarrollo del proyecto se ha llevado a cabo un proceso de evaluación formativa y compartida (Figura 2) en relación a los siguientes aspectos:

1) Funcionamiento del trabajo en grupo

Durante el proyecto es necesario que los miembros del grupo evalúen el trabajo que van diseñando y el desarrollo del trabajo en grupo, por ello, se han realizado autoevaluaciones grupales e individuales en dos sesiones, así como una sesión final dedicada a la evaluación del proyecto en su conjunto.

Además de la observación docente del trabajo grupal en las clases presenciales, con el objetivo de valorar el funcionamiento en grupo, cada equipo de trabajo debe cumplimentar y entregar un acta de trabajo en grupo cada vez que se reúnan para trabajar. 
2) Blog como portfolio

Esta actividad se evalúa mediante un proceso de evaluación compartida incluyendo una autoevaluación grupal, la coevaluación por parte de otros dos grupos de trabajo y la evaluación docente. Para facilitar este proceso, se proporciona una rúbrica con los criterios comunes a evaluar.

3) Proyecto final

El resultado final de cada grupo de trabajo también se evalúa de forma compartida implicando a estudiantes y docentes en el proceso mediante autoevaluaciones y coevaluaciones y utilizando una rúbrica que valora el producto final, así como otra que permite evaluar la defensa oral del mismo. Por su parte, el docente evaluará tanto la defensa oral como el proyecto final.

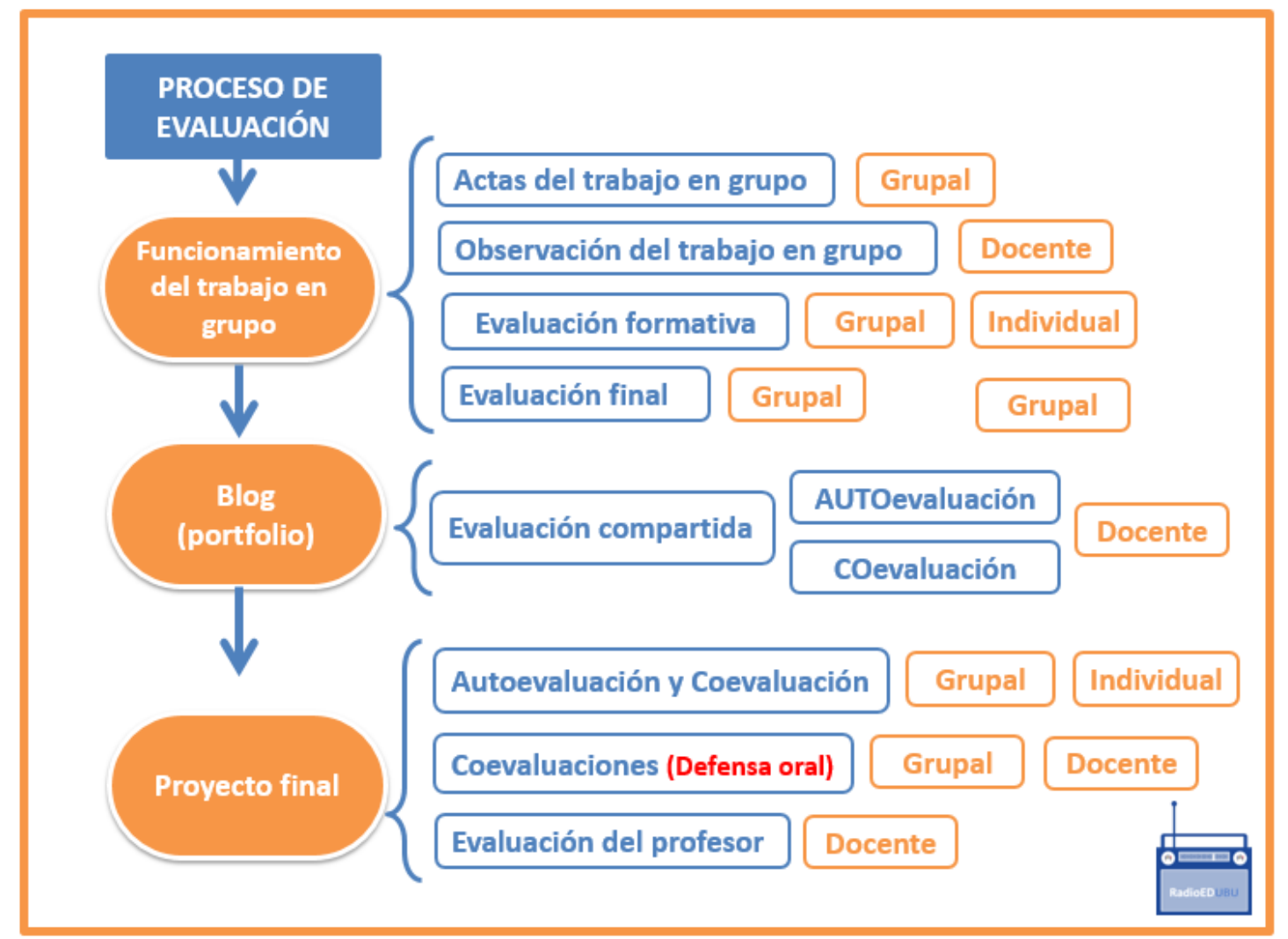

Figura 2.

Proceso de evaluación del proyecto \#RadioEDUBU

\section{Conclusiones}

Las aulas universitarias de la actualidad deben responder a las necesidades de los estudiantes, ya pertenecientes a la denominada Generación App (Gardner y Davis, 
2014), quienes requieren nuevas estrategias de enseñanza que respondan a sus requerimientos de aprendizaje. En este sentido, la utilización de metodologías activas como el ABP permiten a los estudiantes involucrarse en su propio aprendizaje, así como participar en los procesos de evaluación.

Los estudiantes participantes en la experiencia manifiestan que la evaluación formativa y compartida les ha permitido aprender y reflexionar sobre el trabajo realizado, consiguiendo una mayor implicación hacia los objetivos de aprendizaje.

\section{Referencias}

Ausín, V., Abella, V., Delgado, V. \& Hortigüela, D. (2016). Aprendizaje Basado en Proyectos a través de las TIC. Una Experiencia de Innovación Docente desde las Aulas Universitarias. Formación Universitaria, 9(3), 31-38.

Gardner, H. \& Davis, K. (2014), La generación APP: Cómo los jóvenes gestionan su identidad, su privacidad y su imaginación en el mundo digital. Barcelona: Espasa Libros.

Grahame, S. D. (2011). Science education in a rapidly changing world. New York: Hauppauge.

Morales, C., \& Torres, A. (2015). Aprendizaje basado en proyectos para el desarrollo de competencias. Revista Iberoamericana de Producción Académica y Gestión Educativa, 2, 1-10.

Xu, Y. \& WQ Liu (2010). A project-based learning approach a case study in China. Asia Pacific Education Review, (3), 636-370. 